\title{
La educación ambiental en las instituciones de educación superior públicas acreditadas en Colombia*
}

DOI: http://dx.doi.org/10.21830/19006586.178

Recibido: 7 de febrero de 2016 • Aceptado: 5 de junio de 2017

Environmental Education in Accredited Public Institutions of Higher Education in Colombia

Léducation environnementale dans les établissements publiques de l'enseignement supérieur agréés en Colombie

A educação ambiental em instituições de educação

superior públicas acreditadas na Colômbia

Norma Constanza Berdugo Silva ${ }^{a}$
Wendy Yulieth Montaño Renuma

* Artículo de investigación resultado del proyecto de investigación HUM 1802 "Educación Ambiental en las Instituciones de Educación Superior Acreditadas en Colombia”, financiado por la Vicerrectoría de Investigaciones de la Universidad Militar Nueva Granada.

a Universidad Militar Nueva Granada, Bogotá, Colombia. Doctorando en Educación, Universidad Baja California, México. <norma.berdugo@unimilitar.edu.co>.

b Universidad Militar Nueva Granada, Bogotá, Colombia. Trabajadora social, Fundación Universitaria Monserrate. <wendy2909@gmail.com>. 
Resumen. La educación ambiental ha tomado relevancia en las Instituciones de Educación Superior (IES) como un factor importante en el desarrollo de acciones sustentables, dado que despierta una conciencia racional en los sujetos sobre sus actos y su relación con el medio ambiente. La formación de esta conciencia lleva a generar grandes procesos reflexivos y de cambio en la cotidianidad del hombre, y lo invita a repensar sus actos para disminuir la contaminación y los daños producidos por las malas prácticas medioambientales. En este artículo se aborda específicamente el caso de las IES públicas acreditas que han implementado la educación ambiental, con el objetivo de establecer cuáles son los mecanismos y las estrategias que utilizan para desarrollarla, la manera como la conciben y la introducen a su filosofía institucional y las problemáticas que abordan.

Palabras clave: educación ambiental; estrategias; filosofía institucional; instituciones de educación superior; mecanismos y problemáticas.

Abstract. The article aims to know the forms of application of environmental education in public higher education institutions accredited in Colombia to reflect on the management of the realization of these entities in environmental issues and their impacts. The study based on a quantitative approach, empirical-analytical paradigm, and a type of descriptive-transversal research. It found that $47 \%$ of the universities use the strategy to implement environmental education. The articulation of the curriculum is especially in the undergraduate and postgraduate programs. It was also recognized the importance of the use of participatory mechanisms in these institutions for the implementation of this theme by the characteristics of its community.

Keywords: environmental education; higher education institutions; institutional philosophy; mechanisms and problems; strategies.

Résumé. Cet article a pour but de connaître les moyens de mettre en œuvre l'éducation environnementale dans les établissements d'enseignement supérieur public en Colombie accrédités pour réfléchir sur les entités de gestion qui exécutent ces questions et les impacts environnementaux. L'étude a été basée sur une approche quantitative, modèle empirique analytique, de type descriptif de la recherche - transversal. On a constaté que $47 \%$ des universités utilisent comme stratégie pour mettre en œuvre l'éducation environnementale un programme commun en particulier dans le premier et deuxième cycle aussi l'importance d'utiliser des mécanismes de participation dans ces institutions qui mettent en œuvre reconnu thème par les caractéristiques de leur communauté.

Mots-clés: éducation environnementale; établissements d'enseignement supérieur; mécanismes et problèmes; philosophie institutionnelle; stratégies.

Resumo. Este artigo busca conhecer as formas de implementar a educação ambiental no ensino superior público acreditadas na Colômbia para refletir sobre as entidades executam a gestão dessas questôes e impactos ambientais. O estudo foi baseado em uma abordagem quantitativa, paradigma empírico-analítica, tipo da pesquisa descritivo-transversal. Verificou-se que $47 \%$ das universidades usam como estratégia para implementar a educação ambiental a articulação dos currículos especialmente em cursos de graduação e pós-graduação, além disso também reconheceram a importância do uso de mecanismos participativos nessas instituiçôes para implementar esta temática pelas características de sua comunidade.

Palavras chave: educação ambiental; estratégias; filosofia institucional; instituiçóes de ensino superior; mecanismos e questôes. 


\section{Introducción}

El cuidado ambiental es uno de los temas tratados en la actualidad por los diferentes sistemas que componen la sociedad, pues los estudios de diferentes instituciones reconocen que "las consecuencias de la degradación ambiental son producto de múltiples factores que reproducen daños anónimos" (Programa de las Naciones Unidas para el Medio Ambiente [Pnuma], 2006. p. 39). Esto lleva a pensar que, efectivamente, existen acciones que generan daños sobre el ambiente.

De acuerdo con lo anterior, en el siguiente documento se aborda la aplicación de la educación ambiental en las Instituciones de Educación Superior (IES), pues esta es una de las herramientas que permiten al sistema educativo aportar en la prevención y solución de los daños ambientales. Además, dado el alto nivel de responsabilidad que tienen estas instituciones, y considerando que deben llevar a cabo acciones de desarrollo sostenible (Chacón, Montbrun \& Rastelli, 2009) para promover el cuidado y la protección del ambiente, en este documento se identifican las estrategias y los mecanismos que utilizan para desarrollar esta temática, así como las acciones concretas que realizan.

La ruta metodológica se fundamentó en el paradigma empírico-analítico, de enfoque cualitativo, con una investigación descriptiva de tipo transversal debido al bajo volumen de instituciones que se consultaron (Bernal, 2006, p. 123), catorce en total, las cuales cumplían con las características elegidas para la investigación. Esta metodología permitió conocer la aplicación de la educación ambiental en las IES, pues se recolectaron los datos concretos de una manera objetiva y luego fueron sistematizados y triangulados.

De igual forma, en el desarrollo de la investigación se hizo, en primera instancia, una revisión documental para identificar los antecedentes del tema, lo cual dio paso a la descripción del problema de investigación y a la elaboración de instrumentos de recolección de información. Posteriormente, el instrumento fue enviado a los correos de las áreas encargadas de desarrollar los procesos de educación ambiental en las universidades, luego se sistematizaron los datos recolectados en las encuestas y se triangularon.

Finalmente, respecto a la metodología, se debe mencionar que este trabajo se hace desde la perspectiva de trabajo social.

\section{Generalidades}

La implementación de la educación ambiental en las universidades ha sido un proceso que ha llevado varios ańos, pero que principalmente se ha incorporado a las IES a partir de la Conferencia de Tiblisi, "pues en esta se establece que las universidades en su proceso educativo e investigativo deben incluir la educación ambiental” (Ávila, s. f., p. 3), en cuanto son uno de los sistemas que consolidan los conocimientos de los sujetos y movilizan en gran parte sus acciones cotidianas.

Tomando lo anterior como referente, es necesario reconocer que hoy en día varias de estas acciones ambientales se han convertido en una normativa para las instituciones de educación, ya que esto es exigido por las diferentes entidades estatales. Sin embargo, esto solo es obligatorio para las instituciones de educación primaria y secundaria, las cuales deben desarrollar "proyectos 
ambientales, también llamados PRAE” (Rosario, 2012, p. 238), a diferencia de las IES, que lo hacen de manera libre y voluntaria debido a que tienen gran responsabilidad con participar en la educación ambiental impulsando "el mejoramiento de la comprensión y la generación de una conciencia pública sobre la sostenibilidad” (Gacel-Ávila \& Orellana, 2013, s. p.). Por esta razón, las IES públicas acreditadas aplican la educación ambiental de diferentes maneras, con estrategias y mecanismos acordes con las características de su población y filosofía institucional.

Pero antes de describir cómo estas instituciones implementan la educación ambiental, es importante conocer la manera como la conciben. En el estudio realizado se identificó que de las catorce instituciones encuestadas, solo el 79\% enfocan la educación ambiental en el desarrollo sostenible y consideran que se debe aplicar en los diferentes tipos de educación. Esto significa que la mayoría de las universidades encuestadas ven la educación ambiental como un elemento integral y multidisciplinar, que requiere no solo potenciar las habilidades cognitivas de los sujetos, sino que también demanda que sean utilizadas todas las herramientas educativas para lograr un desarrollo sostenible sin poner en riesgo la estabilidad del "medio biofísico" (Manga, 2005. p 39).

$\mathrm{Al}$ respecto, el Ministerio de Ambiente y Desarrollo Sostenible establece que las instituciones deben concebir la educación ambiental como

$[\mathrm{u}] \mathrm{n}$ proceso que le permite al individuo comprender las relaciones de interdependencia con su entorno, a partir del conocimiento reflexivo y crítico de su realidad biofísica, social, política, económica y cultural para que, a partir de la apropiación de la realidad concreta, se puedan generar en él y en su comunidad actitudes de valoración y respeto por el ambiente. (Ministerio de Ambiente y Desarrollo Sostenible, 2002. pp. 18-19)

De igual forma, esto permite que la educación ambiental no solo se enfoque en una temática ambientalista, sino que además aborde de manera global e integrada otros aspectos de la sociedad para comprender así cómo los daños ambientales están afectando al sector político, económico, cultural y social. Asimismo, esta perspectiva hace que el sujeto comprenda que cuidar al medio biofísico genera múltiples beneficios por cuanto previene el desarrollo de otra serie de problemáticas, y le da la oportunidad para "reflexionar críticamente en torno al concepto de formación integral, para detectar avances, logros y dificultades en este aspecto” (Ministerio de Educación Nacional de Colombia [MEN], 1996, s. p.).

Sin embargo, se identificó que el 64\% de las instituciones encuestadas incorpora la educación ambiental en la filosofía institucional de forma desagregada, pues solo la articula con uno de los lineamientos de la institución, ya sea con la misión, la visión, los objetivos, las políticas de calidad, entre otros, mientras que el 36\% sí la articula en cada uno de los componentes de su filosofía institucional: en los objetivos, la misión, la visión y las políticas de calidad/políticas de gestión ambiental. Esta última forma de integración representa una mayor posibilidad de abordar la educación ambiental de manera constante y de lograr efectos positivos sobre el medio ambiente y las diferentes comunidades. Tal idea es sustentada por María Bravo (2003), quien expresa al respecto:

La reconversión ambiental de las IES es una necesidad que hoy debe atenderse de manera prioritaria.

Este cambio se requiere en sus diferentes planos de constitución: en su misión y visión, en toda la 
gama de sus funciones centrales: enseñanza, investigación y difusión; en todas las áreas de conocimiento; en todos los niveles de formación; e involucrando a toda la comunidad educativa educativa.

(Bravo, 2003, p. 2)

Es decir que el componente ambiental, o la educación ambiental, debe estar inmerso en la filosofía institucional, debido a que encamina las acciones de la institución hacia "los objetivos transversales de la educación" (Rengifo, Quitiaquez \& Mora, 2012, p. 6), de manera que esta realice un trabajo continuo e integrado que genere impactos positivos en el medio biofísico. Asimismo, esto le permite esclarecer y demostrar sus líneas de acción frente al tema medioambiental, así como crear y planear estrategias y mecanismos que la lleven a desarrollar la educación ambiental dentro de la comunidad.

Antes de hacer referencia a las estrategias y mecanismos de la IES, primero es necesario identificar en qué consiste cada uno de ellos y de qué manera se articulan. Se entiende por estrategia aquel "programa general de acción que llevan consigo compromisos de énfasis y recursos para poner en práctica una misión básica” (Ronda 2002, s. p.), en tanto que los mecanismos son definidos como el

[c] onjunto de conceptos, declaraciones y explicaciones originadas en la vida cotidiana, en el curso de las comunicaciones interindividuales. Equivalen, en nuestra sociedad, a los mitos y sistemas de creencias de las sociedades tradicionales; puede, incluso, afirmarse que son la versión contemporánea del sentido común. (Moscovici, 1984, p. 181)

Es decir, si bien los mecanismos y las estrategias son herramientas para las universidades, los mecanismos son específicamente las acciones concretas que les permiten ejecutar las estrategias planificadas para aplicar la educación ambiental dentro de las comunidades académicas.

Partiendo de lo anterior, en la información recolectada se identificó que el $47 \%$ de las universidades utilizan como estrategia la articulación de la educación ambiental al currículo de los programas de pregrado y posgrado. Respecto a los mecanismos para cumplir con la estrategia, el 20\% expresa que hace capacitaciones sobre la gestión ambiental y/o prácticas sostenibles; el $29 \%$ desarrolla estrategias dentro de las actividades extracurriculares, haciendo uso de mecanismos como encuentros o conversatorios en torno a temas ambientales en el 13\%; el 12\% orienta sus estrategias en acciones que generen sustentabilidad y sostenibilidad, recurriendo también a mecanismos como capacitaciones que permitan desarrollar estas prácticas; finalmente, el 12\% restante la aplica dentro de los Praus (Proyectos Ambientales Universitarios) y a través de acciones con otras instituciones educativas a nivel nacional, con las cuales desarrolla proyectos de manera conjunta. En este último caso, el 13\% de las universidades recurren a mecanismos tales como prácticas ambientalistas dentro y fuera de la institución, en tanto que el 54\% desarrolla de manera integral capacitaciones, encuentros o conversatorios y prácticas dentro o fuera de sus campus.

Esta interacción entre los mecanismos y las estrategias significa que las universidades llevan a cabo acciones y actividades institucionales e interinstitucionales para desarrollar la educación ambiental dentro de la comunidad académica. Sin embargo, es importante anotar que las instituciones deben hacer un estudio riguroso de la población y sus características, ya que esto les permite detallar una situación específica para formular alternativas y soluciones tentativas (Doorman et 
al., 1991, p. 44). En tal sentido, el estudio de la población se realiza para determinar las estrategias o mecanismos que estén más acordes con el grupo, la institución y con el tema que se quiera aplicar, pues, en la mayoría de los casos, esto determina la efectividad de las acciones.

Algunas de las universidades públicas acreditadas emplean las estrategias y los mecanismos mencionados para abordar diferentes problemáticas. En el estudio se identificó que el $93 \%$ de las instituciones encuestadas tratan en sus procesos de educación ambiental diferentes problemáticas, tales como el mal manejo de las basuras (separación según su composición), emisión de elementos nocivos para el ambiente, contaminación de vertimientos o fuentes hídricas, mala utilización de los recursos, extinción de la biodiversidad, deforestación y explotación de recursos no renovables. Por su parte, el $7 \%$ restante expresa que solamente trata la problemática de la contaminación de vertimientos y fuentes hídricas. Los resultados muestran que gran parte de las universidades públicas acreditadas abordan diferentes problemáticas ambientales, lo cual contribuye aún más a la solución y prevención integrales de los daños ambientales, y además genera un desarrollo sostenible en la comunidad académica.

Estos hallazgos resaltan la importancia de que las IES traten las problemáticas ambientales de manera integral, pues son varios los daños que se producen diariamente en el entorno y que afectan a los diferentes sistemas. De igual manera, se encontró que algunas veces las universidades públicas acreditadas recurren al trabajo en red —entendido como un "sistema de elementos interconectados [...], una forma de trabajar socialmente, de colaborar, de compartir" (Ceballos, s. f., s. p.) - , es decir, conforman redes interinstitucionales con entidades públicas y privadas expertas en el tema para generar acciones y actividades más complejas e integrales.

En los datos recolectados también se reconoció que las universidades recuren a entidades tales como la Secretaría Distrital de Ambiente (SDA), el Ministerio de Ambiente y Desarrollo Sostenible, la Corporación Autónoma Regional (CAR), la Alianza de Redes Iberoamericanas de Universidades por la Sustentabilidad y el Ambiente (Ariusa), la Revista Iberoamericana Universitaria en Ambiente, Sociedad y Sustentabilidad (Ambiens), el Instituto Humboldt, la Red Ambiental de Universidades Colombianas (RAUS), redes de estudiantes de Ingeniería Ambiental y otras universidades, lo cual evidencia que hay una red integral entre instituciones que se preocupan principalmente por promover procesos de educación ambiental para modificar varios elementos que pueden contribuir a la prevención y solución de daños ambientales.

Según la información recogida, las acciones de educación ambiental han tenido efectos positivos: el $31 \%$ en el mejoramiento del manejo de los recursos (agua, elementos reutilizables, entre otros); el $31 \%$ de las universidades expresan que han aumentado la cultura ciudadana; el 23\% ha conseguido mayor sensibilización frente a los efectos nocivos que pueden generar las acciones tomadas por el ser humano y, finalmente, el 15\% ha generado ambientes sanos y sostenibles. Esto muestra lo importante que han sido las acciones de las IES en temas ambientales, pues han generado en pequeña o gran medida impactos positivos sobre la población y el medio biofísico circundante, con lo cual han contribuido al desarrollo de acciones sustentables que permiten prevenir y solucionar dańos en el ambiente.

Aun así, es importante seńalar que los impactos generados a través de la implementación de la educación ambiental se deben a la participación de los diferentes actores implicados en el proceso en cuanto agentes de cambio encargados de "introducir o efectuar a propósito cambios 
en la conducta de los individuos" (Arce, 1963, p. 7). Estos agentes ejecutan las fases del proceso con metodologías como el estudio, el diagnóstico, la planeación, la ejecución y la evaluación, para poder llevar a cabo acciones con la comunidad. Asimismo, también se debe tener en cuenta la participación de los beneficiarios, quienes son "personas a las que se les destina un proyecto, también denominado población objetivo" (Cohen \& Franco, 2006, p. 91), y son considerados como sujetos que participan en las acciones de la educación ambiental, pero de una manera receptiva.

Entre tanto, la información recolectada permitió establecer un panorama general sobre los actores que se benefician en la aplicación de la educación ambiental: el 72\% son estudiantes, el 14\% trabajadores del área administrativa, el 7\% de la muestra expresa que aplica la educación ambiental únicamente con los trabajadores del área administrativa y, finalmente, el otro 7\% la realiza con los docentes.

Es decir que la mayoría de las instituciones no aplica la educación ambiental con cada uno de los actores que componen a la comunidad académica, sino que por el contrario la desarrolla de manera centralizada, dependiendo de los objetivos que tenga la institución con los temas ambientales y lo que quiera lograr. En los resultados se identificó que los principales actores que reciben la educación ambiental son el estudiantado, seguido del área administrativa.

Al respecto, el trabajo de Peńa (2013) da elementos para analizar estos hallazgos, debido a que él expresa que el dańo ambiental es una situación que no solo les concierne a unos sistemas, sino que es una problemática que atańe a cada uno de los sectores y sujetos que componen a la sociedad:

El daño ambiental, por sus propias características, requiere de un tratamiento distinto por parte del instituto civil de la responsabilidad y el de la prescripción, ya que la incerteza es inherente a la cuestión ambiental. Los daños ocasionados al ambiente, en muchas ocasiones, no son consecuencia de una sola acción, sino que son producto de todo un proceso extendido en el tiempo y en el espacio, sin respetar límites o fronteras políticas ni geográficas. (Peña, 2013, p. 117)

Siguiendo esta definición, se puede afirmar que las universidades aplican la educación ambiental con cada uno de los actores que componen a la comunidad educativa porque esta contribuye a la prevención y solución de los daños ambientales, así como porque a través de este tipo de educación se enseña a los sujetos a ejecutar acciones responsables y sustentables con el medio ambiente. De acuerdo con el análisis de la información recogida, se estableció que el 86\% de los actores inmersos participa por razones voluntarias y por lo establecido en las políticas o normatividades institucionales, mientras que el $14 \%$ lo hace únicamente por razones voluntarias. $\mathrm{Al}$ respecto se debe anotar que el hecho de que la mayoría de los actores que participan en el tema de la educación ambiental lo hagan de manera voluntaria favorece los procesos de enseñanza, pues hace de las acciones algo más libre y democrático, en donde las personas entran de manera voluntaria sin ningún tipo de presión, lo cual facilita la adquisición de conocimientos.

Ahora bien, para continuar con la explicación de los actores inmersos se hace necesario identificar las áreas encargadas de implementar la educación ambiental, es decir, aquellas en las cuales se encuentran los agentes de cambio. Teniendo en cuenta el estudio realizado en las IES públicas acreditadas, se identificó que el 34\% ejecuta las acciones educativas ambientales desde las oficinas de procesos institucionales; el 22\% las desarrolla desde la coordinación académica; el 11\% las 
realiza desde el área de responsabilidad social de la universidad; el 11\% desde la oficina de salud ocupacional y gestión ambiental; el otro $11 \%$ desde el instituto de estudios y servicios ambientales de la institución, y, finalmente, el 11\% restante expresa que todas las áreas de la institución son las encargadas de su aplicación.

Lo anterior significa que la mayoría de las universidades tienen un área específica que se encarga del proceso de educación ambiental dentro de la comunidad educativa, mientras que solo el $11 \%$ ven la educación ambiental como un tema que debe ser manejado y abordado por las diferentes áreas de la universidad. Esto lleva a reconocer que en la mayoría de las IES hay una preocupación por contribuir a la solución y prevención de los daños ambientales, a través de la destinación de un área o varias para abordar los temas ambientales. Asimismo, estas áreas ejecutan diferentes acciones haciendo uso de recursos materiales, también conocidos como "bienes tangibles" (Luyo, 2013, s. p.), que facilitan la implementación de la educación ambiental.

En cuanto a la información obtenida, se reconoce que el 31\% de las IES utilizan posters para difundir sus estrategias de educación ambiental; el 22\% usa los recursos digitales y tecnológicos; el 16\% no realiza difusión de sus estrategias; el otro 16\% expresa que emplea todos los recursos posibles, tales como medios tecnológicos, posters e información reproducida a través de eventos, y, finalmente, el $15 \%$ restante solamente recurre a eventos para reproducir la información sobre sus acciones ambientalistas. Con base en esta información se puede afirmar que el $84 \%$ de las universidades se preocupan por informar a su comunidad sobre las acciones que están ejecutando, lo cual representa un beneficio para su desarrollo en cuanto constituye un llamado a la participación activa de las personas para contribuir a la solución y prevención de los problemas ambientales, y en esa medida puede incrementar el número de personas que se ven involucradas en el desarrollo de las diferentes actividades que realizan las IES. Por su parte, el 16\% restante de las universidades no llevan a cabo ningún tipo de difusión, lo cual puede producir el efecto contrario de lo ya mencionado, pues a diferencia de las universidades que informan a su comunidad sobre las diferentes estrategias y mecanismos que utilizan, en este caso puede variar la participación de los sujetos.

Finalmente, se encontró que las universidades utilizan diversos medios de difusión de información, claro está, teniendo en cuenta las características de la población destinataria. Respecto al principal objetivo de los medios de difusión y su definición, Amman (2007) sostiene que "son medios unidireccionales que se complementan con las tecnologías de comunicación-información y forman una red de información y comunicación" (p. 1). Por ende, en el caso particular de las IES, los medios de difusión permiten generar comunicación y transmitir información con los diferentes actores de la comunidad académica, de tal forma que representan un elemento de ayuda que incentiva a los diferentes sujetos a participar activamente en los procesos de educación y gestión ambiental dentro de la institución.

\section{Conclusiones}

Para concluir, de manera general se puede afirmar que las IES encuestadas implementan la educación ambiental como un proceso que tiene como fin prevenir y solucionar varios de los daños ambientales, por cuanto produce un cambio en las prácticas cotidianas de los sujetos que los lleva 
a generar acciones responsables y sustentables con el ambiente. Asimismo, se encontró que es desarrollada a través de diferentes tipos de educación, como la formal, la no formal y la informal, la cual permite generar procesos integrales y les da la posibilidad a los sujetos de recibir la educación ambiental de diferentes maneras, no solamente desde una clase magistral.

También se reconoció que las instituciones, de forma conjunta o desagregada, establecen el tema ambiental en los componentes de su filosofía institucional, lo cual puede representar un beneficio para el desarrollo de los procesos de educación ambiental, pues de este modo la institución va a orientar una de sus líneas de acción expresamente a la prevención y solución de los daños ambientales.

Sumado a esto, se identificaron las estrategias y los mecanismos a través de los cuales las IES públicas acreditadas desarrollan la educación ambiental dentro de la comunidad académica, articulando estas dos herramientas para lograr los objetivos ambientales establecidos en la filosofía institucional. Igualmente, se reconoció que las universidades públicas acreditadas usan principalmente estrategias tales como la implementación de este tema en el currículo académico de los distintos programas, ya que es visto como un proceso en el cual los profesionales de diferentes ciencias deben participar activamente en la construcción de ambientes amigables, pero para ello deben tener conocimientos sobre temas ambientales. Asimismo, la educación ambiental es incluida en los espacios extracurriculares de las instituciones, a través de mecanismos tales como capacitaciones, encuentros, entre otros.

En cuanto a la elaboración de las acciones que previenen y solucionan los daños ambientales, se encontró que las instituciones de la muestra seleccionada utilizan el trabajo en red como un recurso que les permite llevar a cabo el cumplimiento de los objetivos de la educación ambiental. También se hace necesario reconocer que las universidades hacen uso del trabajo en red como un elemento que les da la oportunidad de abordar dentro de la educación ambiental diferentes problemáticas, tales como el mal manejo de las basuras (separación según su composición), emisión de elementos nocivos para el ambiente, contaminación de vertimientos o fuentes hídricas, mala utilización de los recursos, extinción de la biodiversidad, deforestación, explotación de recursos no renovables, entre otros. De igual forma, les ayuda a consolidar los procesos de gestión y educación ambiental que se desarrollan dentro de la comunidad académica, pues cuentan con la participación de diferentes expertos.

Finalmente, se concluye que el trabajo social es una de las profesiones que deben estar inmersas en los procesos de educación ambiental dentro de las IES, pues los profesionales de este campo pueden realizar investigaciones sociales mediante las cuales identifiquen las necesidades de la comunidad y sus características para generar acciones de trasformación y cambio, con el fin de lograr un desarrollo social y sostenible. 


\section{Referencias}

1. Arce, A. (1963). Sociología y desarrollo rural. Turrialba, Costa Rica: Instituto Iberoamericano de Ciencias Agrícolas de la OEA.

2. Amman, R. (2007). Medios de difusión, escuela y derechos humanos en México. Ética Net, 4 (6). Recuperado de http://www.ugr.es/-sevimeco/revistaeticanet/numero6/ Ensayos/Medios_de_difusion.pdf

3. Ávila, A. (s. f.). La educación ambiental a nivel superior (Tesis de posgrado). Universidad Autónoma de San Luis Potosí, México.

4. Bernal, C. (2006). Metodología de la investigación. México: Pearson.

5. Bravo, T. (2003). Las instituciones de educación superior se organizan para participar en el cambio ambiental: El complexus. Revista Agua y Desarrollo Sustentable, 8, 5-56.

6. Ceballos, F. (s. f.). 10 razones para trabajar en red [documento en línea]. Recuperado dehttp://webcache.googleusercontent.com/ search?q=cache:ESLQR77MUM8J:cmap.javeriana.edu.co/ servlet/SBReadResourceServlet\%3Frid\%3D1219334049927 _ $114315+\& \mathrm{~cd}=2 \& \mathrm{hl}=$ es\&ct=clnk\&gl=co

7. Cohen, E. \& Franco, R. (2006). Evaluación de proyectos sociales. México: Siglo XXI.

8. Chacón, R., Montbrun, N. \& Rastelli, V. (2009). La educación para la sostenibilidad: rol de las universidades. Argos (Venezuela), 26 (50), 6-79.

9. Doorman, F. et al. (1991). La metodología del diagnóstico en el enfoque "investigación adaptativa": Guía para la ejecución de un diagnóstico con énfasis en el análisis de finca del pequeño productor agropecuario. Costa Rica: Heredia.

10. Gacel-Ávila, J. \& Orellana, N. (2013). Educación superior, gestión, innovación e internacionalización. Perfiles Educativos, 35 (143), 5-223.

11. Luyo,J. (2013). Recursos dela empresa: humanos, materiales, financieros y tecnológicos [diapositivas en línea]. Recuperado de http:// es.sideshare.net/pepelucholuyoluyo/14-va-semana-rh-rf-rm-rt-re
12. Manga, J. (2005). Gestión ambiental urbana. En, A. Escudero (Presidencia), Economía Ambiental. Encuentro realizado en la Universidad del Norte, Barranquilla, Colombia.

13. Ministerio de Ambiente y Desarrollo Sostenible. (2002). Política Nacional de Educación Ambiental SINA [documento en línea]. Recuperado de http://www.mineducacion.gov.co/1759/articles-339975_recurso_1.pdf

14. Ministerio de Educación Nacional de Colombia (MEN). (1996). Lineamientos curriculares. Recuperado de http://www. mineducacion.gov.co/1759/articles-339975_recurso_1.pdf

15. Moscovici, F. (1984). Social Representations. Cambridge University Press.

16. Peña, M. (2013). Daño ambiental y prescripción. Revista Judicial, 109, 3-140.

17. Programa de las Naciones Unidas para el Medio Ambiente (Pnuma). (2006). Valoración del daño ambiental. México: Pnuma. Recuperado de http://webcache.googleusercontent. com/search?q=cache:TcwB309jOc4J:www.pnuma.org/ gobernanza/documentos/Valoracion_Dano_Ambiental.pd$\mathrm{f}+\& \mathrm{~cd}=3 \& \mathrm{hl}=\mathrm{es} \& \mathrm{ct}=\mathrm{clnk} \& \mathrm{gl}=\mathrm{co}$

18. Rengifo, B., Quitiaquez, L. \& Mora, F. (mayo, 2012). La educación ambiental, una estrategia pedagógica que contribuye a la solución de la problemática ambiental en Colombia. En Independencias y Construcción de Estados Nacionales: Poder, Territorializacióny y Socialización, Siglos XIX-XX. XII Coloquio Internacional de Geocrítica llevado a cabo en la Universidad Nacional de Colombia, Bogotá.

19. Ronda, G. (2002). Concepto de estrategia [información en página web]. Gestiopolis. Recuperado de http://www. gestiopolis.com/un-concepto-de-estrategia/

20. Rosario, V. (2012). Casos de investigación e innovación: procesos para la transformación de las prácticas e instituciones educativas. Bloomington, ID: Instituto para el Desarrollo de la Investigación e Innovación Educativa en Iberoamérica. 